\title{
Tangence
}

\section{Ferrante Palla}

\section{Un poète à la cour de Parme}

\section{Benoît Denis}

Numéro 57, mai 1998

Littérateurs atypiques et penseurs irréguliers

URI : https://id.erudit.org/iderudit/025970ar

DOI : https://doi.org/10.7202/025970ar

Aller au sommaire du numéro

\section{Éditeur(s)}

Tangence

ISSN

0226-9554 (imprimé)

1710-0305 (numérique)

Découvrir la revue

Citer cet article

Denis, B. (1998). Ferrante Palla : un poète à la cour de Parme. Tangence, (57),

87-95. https://doi.org/10.7202/025970ar d'utilisation que vous pouvez consulter en ligne.

https://apropos.erudit.org/fr/usagers/politique-dutilisation/ 


\section{Ferrante Palla. Un poète à la cour de Parme Benoît Denis, Université de Liège - GREGES}

En guise d'introduction à cette communication, je voudrais brièvement rappeler la perspective de recherche que propose le GREGES $^{1}$ quant à l'étude des marginalités littéraires. Elle est en quelque manière latérale, puisqu'il s'agit d'examiner la représentation de l'artiste marginal, telle qu'elle apparaît chez les grands romanciers du XIX ${ }^{\mathrm{c}}$ siècle, de Balzac à Proust, en passant par Stendhal, Flaubert ou Zola. Si la présence de personnages de ce type paraît à première vue discrète chez les écrivains ici envisagés, elle n'est que rarement anecdotique ou insignifiante. Évoluant à la périphérie ou en bordure du texte réaliste, l'artiste marginal semble cristalliser sur lui une série de représentations ayant partie liée avec le déclassement et la malédiction, et s'exprimant sous des dehors aussi variés que la velléité, la procrastination, la manie ou la franche pathologie. En cela, cette figure constituerait dans notre hypothèse une représentation fantasmatique que le romancier réaliste projette de lui-même et de sa pratique, une image tout à la fois idéalisée et déniée du statut et de l'identité sociale qu'il se forge au cours du XIx ${ }^{\mathrm{c}}$ siècle.

Ferrante Palla, dans La chartreuse de Parme, constitue un bon exemple du personnage de l'artiste marginal, tel que nous voudrions l'étudier: médecin, il vit dans les bois qui entourent Parme avec une femme qu'il n'aime plus et dont il a cinq enfants. Bandit d'honneur, il ne pratique le vol qu'en vue de subvenir aux besoins de sa famille et qu'aux fins de couvrir les frais d'impression de ses opuscules poétiques et politiques. Il se déclare en effet "tribun du peuple» (p. 379) ${ }^{2}$ et républicain; il a été condamné à mort pour avoir conspiré contre le prince, ce qu'il revendique hautement. Mais il est surtout "le plus grand poète du nord de l'Italie" (p.

1 L.e Groges (Groupe de recherche sur les événements génériques du symbolismo) est actuellement composé de Jacques Dubois, Jeannine Paque, JeanPierre Bertrand et Benoît Denis.

2 L'édition de référence ici utilisée est: Stendhal, La cbartreuse de Parme, Paris, GF-Flammarion, 1964. 
88

382), ce que le texte répète de façon insistante, sans jamais pourtant en donner ne fût-ce que l'esquisse d'une représentation concrète. Amoureux de la Sanseverina, il empoisonnera le prince de Parme et mènera une insurrection populaire - rapidement réprimée par le comte Mosca - avant de s'embarquer finalement pour l'Amérique. Ces caractérisations successives marquent bien l'aspect touffu du personnage, dont l'identité est atypique ou marginale notamment en ce qu'elle emprunte, avec un syncrétisme anarchique, à des modèles variés et à certains égards inconciliables. Les quelques remarques qui suivent voudraient mettre un peu d'ordre dans la représentation qu'on peut se faire du grand poète dont l'image traverse le roman de Stendhal.

Il convient d'abord de noter que Ferrante Palla n'apparaît que de façon discontinue et épisodique dans La chartreuse de Parme. C'est un personnage en quelque sorte météorique, qui n'entre en scène qu'à la fin du roman, au chapitre XXI, lorsque l'essentiel de l'action se trouve déjà consommé. Force est d'ailleurs de reconnaître que Stendhal n'avait guère besoin de Palla pour mener à terme son récit et que l'apport du "grand poète" à l'intrigue est de l'ordre de l'accessoire. Par certains côtés, la présence textuelle de Palla est ainsi à l'image du récit stendhalien dans son entier, dont la narration est primesautière et désinvolte, fonctionnant par accélérations brusques, ruptures et ellipses. Mais il importe surtout de voir que le personnage est très largement gratuit dans l'économie du récit ${ }^{3}$. Rigoureusement non nécessaire, Ferrante Palla est en reste dans La chartreuse, comme s'il s'agissait à la fois d'un personnage injustifié et injustifiable, en tout cas excédentaire et irréductible à l'ordre du récit réaliste.

En second lieu, Ferrante Palla apparaît comme un personnage impensable parce que contradictoire. Le plus grand poète du nord de l'Italie est en effet également un fou: le texte le désigne comme tel à plusieurs reprises et délivre à son sujet un certain nombre de signes qui vont en ce sens; Palla aime par exemple à parler de lui à la troisième personne et tombe à plusieurs reprises dans une sorte de délire prophétique qui a toutes les

3 On pourrait néanmoins avancer que l'utilité narrative de Palla consiste en l'assassinat du prince; il faut cependant bien voir que, dans le roman, une fois Fabrice libéré, la mort de Ranuce-Emest IV perd pour la Sanseverina de son urgence et de son intérêt. En exécutant néanmoins sa mission, Palla accomplit un acte qui a, une fois de plus, perdu toute nécessité. 
apparences de la "folie littéraire " ${ }^{4}$. La description elle-même ne parvient à rendre compte du personnage qu'en multipliant les oppositions et en manifestant un fort taux de redondance, comme si le texte ne pouvait qu'asséner répétitivement cette série de traits contradictoires qui constituent l'identité du personnage:

Il était jeune, fort bel homme, mais horriblement mal mis; ses habits avaient des déchirures d'un pied de long, mais ses yeux respiraient le feu d'une âme ardente. [...] La duchesse avait remarqué qu'il était horriblement maigre; mais ses yeux étaient remplis d'une exaltation si tendre, qu'ils lui ôtèrent l'idée du crime. [...] La duchesse comprit qu'il était un peu fou, mais elle n'eut point peur; elle voyait dans les yeux de cet homme qu'il avait une ame ardente et bonne, et d'ailleurs elle ne haïssait pas les physionomies extraordinaires. (p. 377-378)

La contradiction se noue en Ferrante Palla dans la concomitance du génie et de la folie, comme si la limite qui sépare ces deux états était indiscernable, et que le cas de Palla ne pouvait pas être univoquement rapporté à l'un ou l'autre des termes de l'alternative 5 . Plus généralement, ce personnage total - il est poète, tribun, médecin, bandit d'honneur et grand amoureux est voué à la folie, à l'exil et l'échec en même temps qu'il représente une figure singulièrement triomphante, chez qui la logique du désir reste toujours la plus forte - c'est peut-être là que réside sa folie -, mais forte au point d'intégrer le principe de réalité pour parvenir à l'assouvissement: champion du travestissement et de la débrouille, sa capacité à mener à bien, contre toute attente, ses entreprises délirantes culmine dans l'habile empoisonnement du prince. Et significativement, la Sanseverina, l'autre grand personnage désirant de La cbartreuse, éprouve pour le grand poète fou une fascination qui ressemble un peu à de l'amour, et qui laisse d'ailleurs penser que, jusqu'à un certain point, Palla se dresse en double de Fabrice, réalisant une nature,

4 Voir par exemple, ce message adressé à la Sanseverina: "Le tribun a pris cent francs par mois, non plus; avec le reste on voulut ranimer le feu sacré dans des âmes qui se trouvèrent glacées par l'égoïsme. Le renard est sur meśs traces, c'est pourquoi je n'aj pas cherché à voir une demière fois l'être adoré. Je me suis dit, elle n'aime pas la république, elle qui m’est supéricure par l'esprit autant que par les grâces et la beauté." (p. 434)

5 Ce que marcue bien cette réflexion de la Sanseverina : $\$$ i le ciel voulait cue Ferrante fût devenu tout à fait fou ou manquât de courage, l. .l. (p. 405). En Ferrante Palla, on ne sait trop ce qui l'emporte, du fou ou de "l'homme sublime: 
folle et poétique, présente à l'état de virtualité chez le jeune héros de La chartreuse:

Ferrante! s'écria-t-elle; homme sublime! [...]

Voici le seul homme qui m'ait comprise, se dit-elle, c'est ainsi qu'en eût agi Fabrice, s'il eût pu m'entendre. (p. 385)

Enfin, il importe de marquer chez Palla l'étonnante congruence du poétique et du politique, puisqu'il se présente à la fois comme "le plus grand poète du nord de l'Italie" et " tribun du peuple.. La chartreuse de Parme, qui est écrite sous la Monarchie de juillet mais dont l'action se déroule sous la Restauration et dans une Italie sous tutelle autrichienne, met en scène la rivalité qui oppose conservateurs et libéraux à l'intérieur du régime absolutiste du duché de Parme. Face à ces deux partis, Ferrante Palla occupe visiblement une position tierce, celle qui correspond au républicanisme et à un socialisme utopisant dont le lieu possible de concrétisation serait l'Amérique. Au-delà cependant de cette distribution politique des rôles dans le roman et de ses référents historiques possibles (légitimisme, libéralisme et socialisme utopique français; absolutisme et carbonarisme italiens), il faut surtout souligner que la position de Ferrante Palla ne prend sens qu'en fonction de la représentation générale que Stendhal propose du politique dans La chartreuse. Celle-ci est très largement de l'ordre d'un scepticisme désabusé et souriant, en vertu duquel on voit constamment se brouiller les limites et les repères entre les idéologies mises en présence: le comte Mosca est ainsi un ancien combattant napoléonien devenu chef du parti conservateur, ce qui ne l'empêche pas d'apparaître en bien des cas plus libéral dans ses méthodes et dans ses objectifs que ses rivaux groupés autour de la Raversi et du fiscal Rassi. Brièvement dit, Stendhal suggère que le gouvernement produit constamment une relativisation des doctrines politiques, dont le contenu réel s'efface au profit des intrigues et du cynisme qu'exige l'exercice du pouvoir. Ferrante Palla, quant à lui, représente le pôle idéaliste de la politique, lequel ne se conçoit que dans une position d'éternel oppositionnel jouant toujours perdant.

Plus globalement, on connaît la formule fameuse selon laquelle: "La politique dans une ouvre littéraire, c'est un coup de pistolet au milieu d'un concert, quelque chose de grossier et auquel pourtant il n'est pas possible de refuser son attention." (p. 419) La politique paraît ainsì chez Stendhal la malédiction du 
romancier réaliste, ce qu'il ne peut pas passer sous silence et ce qui l'empêche de faire complètement œuvre littéraire. Or Ferrante Palla semble précisément échapper à cette alternative dysphorique. Chez lui, il n'est presque pas possible de faire le départ entre ce qui ressortit au poétique et au politique. L'une et l'autre activités se trouvent constamment confondues, au point d'être indiscernables, comme le révèle cet étrange dialogue entre la duchesse et le fou:

- Quels ouvrages [écrivez-vous]?

- La... aura-t-elle jamais une chambre et un budget?

- Quoi, dit la duchesse étonnée, c'est vous, monsieur, qui êtes l'un des plus grands poètes du siècle, le fameux Ferrante Palla!' (p. 379)

Cette confusion des genres chez Ferrante Palla doit aussi être rapportée à un thème qui traverse $L a$ chartreuse dans son entier: l'opposition irréductible de l'érotique et du politique, en tant que deux modes d'appréhension de l'existence inconciliables parce que contradictoires (la réalisation de l'un entraînant nécessairement le renoncement à l'autre). Ainsi, le comte Mosca apparaît dans le roman comme celui qui politise l'érotique, son amour pour la Sanseverina étant continuellement conditionné par le cynisme des intrigues de cour dans lesquelles sa maîtresse et luimême sont engagés. Ferrante Palla, au contraire, est très remarquablement celui qui érotise le politique, ses prises de position obéissant d'abord à une logique désirante (l'exécration infinie qu'il voue au prince de Parme). Bien plus, la politique chez lui relève du comportement amoureux : parlant de son prochain exil en Amérique, Ferrante écrit à la duchesse: "je verraỉ si je dois encore aimer la seule rivale [la république] que vous ayez dans mon cœur." (p. 434) On s'explique mieux dès lors pourquoi le

6 On notera ici que la folie de Palla devient significativement la «folic" du texte: Palla parle politique et la duchesse lui répond poésie, et c'est à ce moment seulement que cette dernière semble vraiment identifier un Ferrante Palla qui s'est explicitement nommé dès le début du dialogue. Autre exemple de cette confusion entre poétique et politique, cette lettre de Palla et l'interprétation qu'en fait la Sanseverina: "Alors je ferai enterrer, sous le grand buis du jardin que vous remarquâtes une fois en mes jours heureux, une boîte où se trouvent de ces choses qui font calomnier les gens de mon opinion. [...] Puisqu'il a une imprimerie à ses ordres, se dit la duchesse, bientôt nous aurons un recueil de sonnets, Dieu sait le nom qu'il m'y donnera." (p. 434) 
92

poète ne peut passer à l'action et achever son projet politique (le régicide) qu'à partir du moment où cet acte politique devient simultanément serment amoureux envers la duchesse: "Ma joie est de mourir en nuisant au tyran, une bien plus grande joie de mourir pour vous " ${ }^{7}$ (p. 384); ou encore, lorsque la duchesse lui demande de tuer le prince pour satisfaire sa vengeance: "je suis plus fort maintenant! s'écriait ce fou; je n'ai plus de doute sur la légitimité de l'action!» (p. 387)

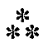

Personnage dont la présence dans l'économie du roman relève de la gratuité pure et qui semble constamment dépasser les antinomies sur lesquelles se construit La chartreuse de Parme (érotique-politique; littérature-politique; folie-génie; etc.), Ferrante Palla apparaît comme une figure limite, un possible littéraire, politique et amoureux qui constituerait l'impensé du roman, c'est-à-dire une manière de repoussoir à travers lequel le récit interroge sa nature et ses limites.

Dans cette perspective, il n'est pas inutile de revenir sur la caractérisation la plus forte du personnage, celle qui en fait "le plus grand poète de ce siècle". Bizarrement en effet, il semble qu'à côté de cette assertion répétée, la représentation du personnage ne fasse jamais droit à cette facette de son identité: le génie poétique de Ferrante Palla constitue une évidence acquise, qui ne peut être qu'affirmée, mais jamais montrée. Comme si Stendhal devait dire et redire qu'un tel génie existe, mais qu'il ne disposait pas des moyens de le représenter. On touche ici à ce qui constitue le coeur de l'exclusion de Palla: s'il est condamné à la folie, à l'exil et à la marginalité, c'est parce que le plus grand poète d'Italie est avant tout irreprésentable. Aussi le récit ne cesse-t-il de l'évacuer de diverses manières. Soit on l'exile dans les bois et les collines qui entourent Parme, puis de façon plus décisive encore dans la lointaine Amérique. Soit on le "récupère" en le détournant de sa mission historique (le soulèvement du peuple et l'instauration de la

7 On notera en passant que Stendhal n'écrit pas "Si ma joie est de mourir en nuisant au tyran, une bien plus grande joie serait de mourir pour vous", comme s'il s'agissait d'atténuer, jusqu'à la limite de la correction syntaxique, la structure oppositive de la phrase afin de suggérer la conciliation la plus totale des impératifs amoureux et politique. 
république) et en le faisant servir une cause politique qui contredit nécessairement la sienne (la Sanseverina et Mosca font du régicide un moyen de changer de souverain afin de rentrer en cour et faire gracier Fabrice). Si, par aventure, il s'agit de faire entrer le tribun dans la ville, c'est-à-dire dans la société, c'est pour le confiner dans un étroit réduit ménagé dans la muraille du palais Sanseverina, qui devient l'image de la condition sociale misérable du poète authentique. Dans La chartreuse de Parme, le grand poète paraît par excellence celui qui ne peut être intégré et dont la condition est impossible et intolérable, à lui comme aux autres.

Ferrante Palla constitue donc une représentation tout à la fois idéalisée et refusée de l'écrivain, tel que sa physionomie sociale se dessine dans la première moitié du xix ${ }^{\mathrm{e}}$ siècle. Figure totale, chez laquelle ce qui relève du littéraire ne se distingue pas a priori de l'amoureux ou du politique, le grand poète est aussi celui qui revendique hautement son indépendance:

C'est peut-être pour cela que j'ai quelque talent. Jusqu'ici tous nos auteurs qui se sont fait connaitre étaient des gens payés par le gouvernement ou par le culte qu'ils voulaient saper. Moi, primo, j'expose ma vie; secundo, songez, madame, aux réflexions qui m'agitent lorsque je vais voler! (p. 379)

Palla est sans doute un fou et un marginal, mais ce qui fait la grandeur de sa folie et de sa marginalité, c'est qu'elle est aussi revendication d'une liberté du créateur qui doit être conquise de haute lutte. À travers le poète de La chartreuse se dessine en effet de façon détournée une représentation idéalisée de l'autonomie du littéraire par rapport au politique, autonomie qui apparaît comme le gage d'une certaine forme d'excellence ${ }^{8}$. Il est surtout remarquable que cet appel à l'indépendance du créateur se conçoive dans le récit stendhalien dans une liaison très forte avec la revendication des libertés démocratiques.

8 Voir par exemple, cette opposition entre deux sonnets, dont le premier, par l'enflure qui semble le caractériser, provient vraisemblablement d'un "médiocre" poète de cour: " Dès le lendemain de l'évasion de Fabrice, plusieurs personnes avaient reçu un sonnet assez médiocre qui célébrait cette fuite comme une des belles actions du siècle, et comparait Fabrice à un ange arrivant sur terre les ailes étendues. Le surlendemain soir, tout Parme répétait un sonnet sublime. C'était le monologue de Fabrice se laissant glisser le long de sa corde, et jugeant les divers incidents de sa vie. Ce sonnet lui donna rang clans l'opinion par deux vers magnifiques, tous les connaisseurs reconnurent le style de Ferrante Palla. (p. 411) 
94

Si la figure de Ferrante Palla est ainsi redevable d'un certain romantisme social, en vertu duquel l'autonomie du poète se donne comme inséparable des libertés politiques, il faut aussi redire que, de multiples façons, le texte tend à marginaliser la figure qu'il construit. Impensable et irreprésentable, le grand poète semble voué, jusque dans son idéal démocratique", à l'échec et à l'exclusion, comme si sa logique désirante se trouvait en porte-à-faux avec celle du monde que le roman prend en charge.

Sartre, distinguant dans Qu'est-ce que la littérature? la poésie de la prose, suggérait que le poète s'engage à perdre là où le prosateur s'engage à réussir ${ }^{10}$. L'intuition sartrienne, sans doute discutable, mais fidèle à la doxa littéraire, permet de rendre compte du cas de Ferrante Palla, ce grand poète irréductible à l'ordre réaliste du roman. Elle suggère en tout cas que la réussite, pour l'écrivain réaliste, consiste en une large part à se soumettre à la positivité du réel et à l'accepter, en censurant tout ce qui relève d'une logique désirante. La conversion au réalisme, et à sa formidable efficacité, suppose ainsi le sacrifice, l'exil ou la récupération du désir et de l'érotique: il faut qu'ils servent et se transforment en autre chose, comme chez Balzac où l'amour et la beauté deviennent les signes sociaux de l'ambition 11. La chartreuse de Parme, dont l'un des motifs centraux est celui de l'enfermement (de Fabrice dans ses prisons successives), ne dit finalement rien d'autre: à travers la confrontation de l'érotique et du politique, il s'y dit constamment, et avec nostalgie, que la réussite ne s'obtient qu'en faisant barrage au désir; faute d'y renoncer, la plupart des protagonistes (Fabrice, Clélia, la Sanseverina), mourront; seul le comte Mosca, grâce à son cynisme de courtisan consommé, et non sans avoir souvent souhaité tout lâcher, parviendra à durer et finira "immensément riche", faisant comparer le gouvernement de Parme "à celui des grands ducs de Toscane". (p. 509)

9 Voir ainsi ce que le narrateur dit de l'Amérique: "D'un autre côté, en Amérique, dans la république, il faut s'ennuyer toute la journée à faire une cour sérieuse aux boutiquiers de la rue, et devenir aussi bête qu'eux; et là, pas d'Opéra." (p. 445)

10 Jean-Paul Sartre, Qu'est-ce que la littêrature?, Paris, Gallimard, coll. "Folioessais", 1985, p. 41-44, note 4.

11 Pour une analyse comparée de Stendhal et de Balzac sur ce point précis, on se reportera par exemple à D. A. Miller, "Corps masculins", dans Denis Hollier (dir.), De la Littérature française, Paris, Bordas, 1993, p. 642-648. 
Ferrante Palla est par excellence celui qui contredit et subvertit l'ordre réaliste du roman, le plie à ses désirs et à sa folie. Son triomphe et son échec sont en cela indissolublement liés, car ce qui rend son personnage magnifique, c'est l'horizon de défaite et d'insatisfaction perpétuelle qu'il s'est choisi. Sa présence dans les marges de La chartreuse indiquerait ainsi que le roman ne peut supporter le véritable écrivain, le grand poète, parce que celui-ci refuse de se soumettre au principe de réalité qui fonde le canon réaliste. La folie de Palla représente l'impensé du roman, cette "folie du texte" que le romancier réaliste doit limiter autant qu'il est en son pouvoir. En cela, il n'est pas étonnant que Palla soit condamné à n'être représenté qu'en bordure du récit, comme une figure excédentaire et improbable: le plus grand poète du nord de l'Italie représente la limite du réalisme et la part de lui-même à laquelle le romancier doit renoncer pour réussir dans son entreprise de conversion au réel.

À travers Ferrante Palla, Stendhal saisit l'écrivain dans un moment où son statut et son identité sociale se modifient de façon décisive. C'est évidemment la modernité littéraire qui est souterrainement à l'œuvre dans le personnage et les contradictions qui l'habitent. Tout ce qui relève chez lui de la confusion entre politique et poétique peut ainsi apparaître comme la survivance nostalgique d'une époque, pas si éloignée, où l'écrivain pouvait encore tout vouloir et vouloir tout écrire, parce que la revendication de son indépendance ne se distinguait pas encore du combat collectif pour les libertés. Stendhal semble ainsi avoir perçu qu'un champ littéraire autonome était en gestation, transformant radicalement la figure de l'écrivain et les conditions d'exercice de sa fonction. En plaçant Ferrante Palla en contrepoint des exigences réalistes du récit, le romancier suggère ainsi que lui-même participe pleinement à l'émergence de ce champ littéraire autonomisé, mais qu'il sait aussi qu'à l'intérieur de ce champ, ce n'est pas lui qui est destiné à occuper la position de plus grande légitimité, mais une figure à certains égards impensable et improbable, celle de Ferrante Palla, le grand poète maudit. 